\title{
A NEW CODING SCHEME FOR 2-D AND 3-D MR IMAGES USING SHAPE ADAPTIVE INTEGER WAVELET TRANSFORM
}

\author{
Ahhishek Mehrotra
}

\author{
Department of Electrical Engg. \\ Indian Institute of Science \\ Bangalore, India \\ mehrotra@ee.iisc.ernet.in
}

\author{
R.Srikanth \\ Department of Electrical Engg. \\ Indian Institute of Science \\ Bangalore, India \\ srikanth@ee.iisc.ernet,in
}

\author{
A.G.Ramakrishnan \\ Department of Electrical Engg. \\ Indian Institute of Science \\ Bangalore, India \\ ramkiag@ee.iisc.ernet.in
}

\begin{abstract}
Wepropose a new Shape Adaptive Integer Wavelet Transform based progressive transmission coding scheme for 2-D and 3-D MRI. The scheme consists of (a) extraction of shape information (b) Shape Adaptive Integer Wavelet transform (c) Intraband Wavelet enoding arid $(d)$ entropy coding. The proposed scheme results in improved performance of progrssive transmission as compared to the conventional rectangular Wavelet transform coding schemes. The main contribution of this paper is its unique approach which rejects the noisy background rather than considering itfor lossy conipression as proposed in most of the recent schemes.This rejection of unwanted information results in considerable decrease in bit rate. Another contribution is object based 3-D coding which is óut of scope of baseline JPEG2000.
\end{abstract}

\section{INTRODUCTION}

Data compression is useful in reducing the storage and/or transmission bandwidth requirements of medical images. Compression methods are broadly classified into reversible (lossless) and irreversible (lossy) methods. In the medical image scenario, lossy compression schemes are not generally used. This is due to a possible loss of useful clinical information which may influence diagnosis. In addition to these reasons, there can be legal issues. Hence there is a need for efficient lossless compression schemes for medical data.

The Discrete Wavelet Transform (DWT) is widely acknowlcdged to feature excellent decorrelation properties but DWT based encoders cannot achieve genuine lossless compression, due to the limited precision of computer arithmetic. A more efficient approach to lossless compression is the use of integer transforms such as Integer Wavelet Transforms. The transform coefficients exhibit the feature of being exactly represented by finite precision numbers and this allows truely lossless coding. Although the use of Integer Wavelet Transforms gives lossless compression, the compression ratios are generally low for perfect reconstruction. In the case of MR images, only $40 \%$ of the image consists of clinically useful information and rest of the portion is the noisy background. This makes MR images ideal for object based coding.
A compression scheme with progressive transmission capability is very useful in applications like telemedicine, where data needs to be transmitted at a faster rate. With this capability, image data can be transmitted from a coarse to finer resolution. The user, at the receiving end can download the image up to the resolution required and if necessary, up to perfect reconstruction.

The agreement of the image processing community on object-based approaches is proved by the fact that the incoming standard for still image compression JPEG2000 features region of interest (ROI) based functionalities [4]-[5]. Nevertheless, the baseline JPEG2000 does not address 3-D data.

In this paper, we present a MRI coder which uses Shape Adaptive Integer Wavelet Transform (SAIWT) and has progressive transmission and lossless reconstruction options. The progressive transmission scheme is based on the scheme proposed by [2] where correlation within the band is exploited as against EZW and SPIHT schemes where correlation across the bands are exploited. The difference between our approach as compared to the approach in [7] is that they consider only the brain portion as the useful information in MR images and suitable for lossless compression, the skull portion and the noisy background may be compressed in a lossy way, whereas our approach completely rejects the undesired, noisy background and reconstructs the image portion losslessly. The approach in [7] takes the rectangular wavelet transform of the whole image (including the noisy background), whereas our approach requires taking Shape Adaptive Integer Wavelet Transform of only the desired portion and rejecting the noisy background. The advantage of our scheme is in its simple implementation and effective performance in terms of bit rate as compared to the conventional rectangular wavelet transform based schemes. The block diagram of the proposed scheme is given in Fig. 1

\section{EXTRACTION OF SHAPE INFORMATION}

A typical MR image consists of two parts:

1. Air part (background)

2. Flesh part (foreground)

The flesh part contains the useful clinical information which needs to be compressed without any loss. On the other hand, the air part does not contain any clinical information. It is 

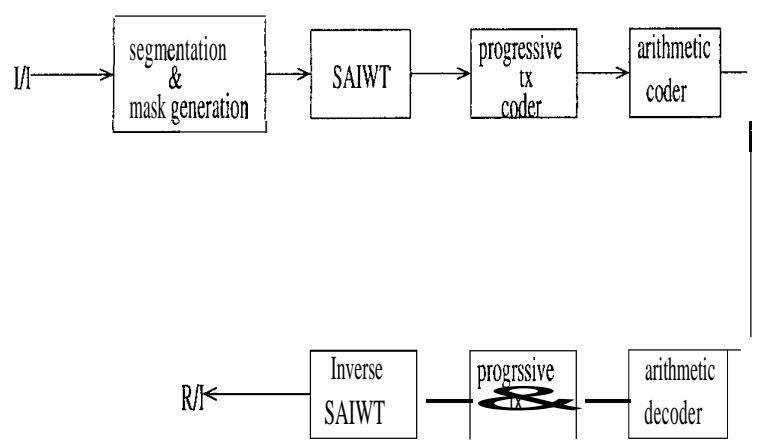

Figure 1: Block Diagram of The Proposed Scheme (SAIWT : Shape Adaptive Integer Wavelet Transform; I/I : Input Image; $R /$ : Reconstructed Image)

only noise and consumes unnecessary bit budget and impairs the performance of a compression scheme. In [1], a scheme is proposed which uses two source models, one for background and the other for foreground, and an improvement in performance is reported. But no justification is given to code the air part as there is no useful information present in it. In this work, we ignore the air part. We generate image masks in such a way that the flesh part is totally included and the pixel values in the air part are made zero. The rest of this section explains an image independent algorithm for mask generation.

Morphological operations can be effectively used to generate image masks, which contain a value of '1' in the foreground and a value of ' 0 ' in the background. The original image is then multiplied with these masks to obtain "background noise-free" images while keeping the information in the foreground intact. Figure 2 shows the generated mask after each morphological operation stated above. Figure 3 shows an MR image, its mask and the image obtained after multiplication with the mask. The algorithm for generating the mask is given below:

1. Binarize the image with a threshold decided by the histogram of the image.

2. Holes may be formed within the foreground. Close these holes using morphological 'closing' operation.

3. Background may contain spurious lines. Use morphological 'erode' operation to remove these lines.

4. The above erosion operation also erodes the boundary of the foreground region. To make sure that the mask spans the entire foreground region, use morphological 'thickening' operation to thicken the boundary of the foreground region. This is followed by bridging operation to bridge the gap inbetween the mouth portion.

5. Multiply the original image with the resulting binary mask. We use this binary mask information for shape adaptive wavelet transform based compression scheme as explaiiied in the following sections.

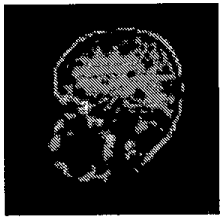

(a)

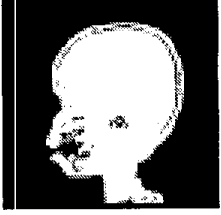

(b)

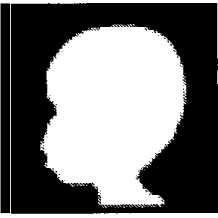

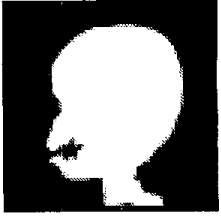

(c)

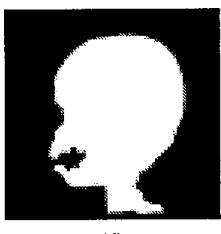

(d)

Figure 2: Mask after various morphological operations. (a) original image (b) mask after binarisation (c) mask after closing operation (d) mask after erosion (e) mask after thickening and bridging.

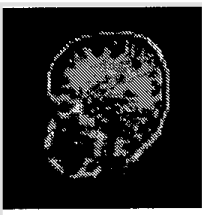

(a)

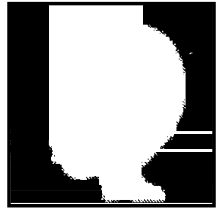

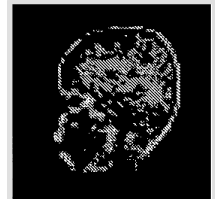

Figure 3: Suppression of background in an MR image using morphological operations. (a) original image (b) the generated mask (c) background suppressed image.

\section{SHAPE ADAPTIVE INTEGER WAVELET TRANSFORM}

We modify the technique given in [10] to obtain integer transform coefficients required for lossless compression. The motivation behind using this scheme is that the wavelet transform is performed faster and with easier boundary extension. One more advantage of this scherne over the scheme used in [7] is that in this scheme there is no need to code extra coefficients to avoid artifacts along the region boundaries. This 1-D transform is applied to each row and then to each column to obtain 2-D transform as follows:

Step 1: Row Transform: Each row intersects the object and forms one or more foreground segments which can be found from the shape information present in the mask. A lifting wavelet with a symmetrical boundary extension is applied on each segment independently. We always align the center of the low pass wavelet filter with even index $2 \mathrm{i}$ and align the center of the high pass filter with odd index $2 i+1$ with index position calculated from the start of the segment. If there is no object pixel at $2 \mathrm{i}$ or $2 \mathrm{i}+1$ there will be no corresponding coefficient at index $\mathrm{i}$ of the low-pass or high-pass bands. 
Suppose a segment of length 8 pixels is fed into the lifting wavelet module as shown in Fig 4(a). We furthur assume that the low pass wavelet filter is aligned with coefficient $x_{0}$. The biorthogonal 9-7 lifting wavelet [9] can be depicted by a 4-stage lifting with coefficients $\mathrm{a}=-1.586, \mathrm{~b}=-0.052, \mathrm{c}=$ 0.883 and $\mathrm{d}=0.444$. The wavelet coefficients are calculated as follows. First high pass filtering is performed which updates the coefficients at odd indices and then a low-pass filtering is performed which updates the coefficients at even indices. The third and fourth stages are high and low-pass stages respectively, which generate the final high and low-pass coefficients. The entire forward lifting operation is shown in Fig 4(a). A basic element of lifting can be described by the equation given below and is also illustrated in Fig 4(b).

$Y=([L+R) * d]+X$

$\mathrm{X}, \mathrm{Y}$ coefficients before and after lifting

$L, R$ : left and right neighbouring coefficients

d: lifting parameter of the current stage

[]: floor operation

Step 2: Column Transform: The low and high-pass bands of horizontal transform are further processed column by column. Each column intersects the object and forms one or more vertical segments. We perform the procedure same as described above and align the low-pass filter with even index $2 \mathrm{j}$ and high-pass filter with odd index $2 \mathrm{j}+1$. The resulting coefficients are stored in the index $j$ of the low and high pass vertical bands respectively. The number of wavelet coefficients after SAIWT is exactly the same as the number of pixels in the object

Decomposition of the mask: When we apply row transform to the MR image we get low pass and high pass image. Simultaneously we generate two binary masks, one for low pass image and the other for high pass image by making the regions where we get transformed coefficients as ' 1 ' and other reigions as '0'. Similarly, from these two masks we generate masks representing $\mathrm{ll}, \mathrm{h}, \mathrm{hl}, \mathrm{hh}$ images by applying column transform to low and high pass images. Using the above method the 11 mask can be again decomposed along with the 11 image to get the 2-level decomposed mask.

Fig 5 shows the 2-level 2-D shape adaptive transform of a MR slice along with the decomposition of mask. At each level, the decomposed mask is used for the next level decomposition. Decomposed mask in each band also gives the object's shape in that band. This information is used for encoding the wavelet coefficients as explained in the following section.

\section{CODING SCHEME}

We modify the coding scheme proposed in [2] for shape adaptive coding. This scheme exploits correlation within the band whereas the schemes proposed by [6] and [3] exploit interband correlation using zerotrees. We also reduce the side information required to be sent to the decoder using the idea proposed in [8]. The details of the scheme are given below:

1. Apply n-level 2-D separable shape adaptive integer wavelet transform to the given image.

2. Assign the label $d n c$ (do not care) to wavelet coefficients which do not belong to the object using the mask information shown in Fig 5.

3. Partition the image into $v \times v$ lattices.

4. For each lattice $k$, find the maximum absolute value $w_{\max }$ without considering coefficients with label $d n c$. Let $T_{k}=\left|\log _{2} a b s\left(w_{\text {max }}\right)\right|$ be the threshold of the $k t h$ lattice. Store these values in the array $t h$.

5. Initialize an array lstb of size equal to that of $t h$.

6. Set the maximum of all the thresholds as the global threshold, $T$,.

7. Scan the wavelet image starting from the lowest frequency band to the highest frequency band in zig zag manner. In each band, the lattices are scanned in raster order.

8. If $T_{k}<T$, the lattice is insignificant with respect to $T_{9}$ and no information is sent to the decoder. A binary ' 0 ' is stored in $l s t b$. If $T_{k}>=T$, the lattice is significant and needs to be transmitted to the decoder. If this lattice is first time significant, a ' 1 ' is recorded in the list $l s t b$. If the lattice is already significant, no information is sent to the decoder, since this lattice will also be significant for the future lower global thresholds.

9. If the lattice is significant, check for the significance of each coefficient in raster scan order. If the coefficient is absolute significant, a ' 1 ' is appended to the significant list lis 1 otherwise a ' 0 ' is appended. If the coefficient is positive significant, a ' $O$ ' is appended to the sign list lis 2 or a ' 1 ', if it is negative significant.

10. From the second pass onwards, those coefficients which are already significant in the previous passes, are refined by sending the next MSB in the list ref.

11. After all lattices are scanned, set $T_{g}=T_{g}-1$.If $T_{g}>=$ 0 go to step 7, otherwise stop.

The lists lis1, lis 2, 1stb and ref can be further losslessly compressed by employing arithmetic coding. Since the most important coefficients (with higher thresholds $T_{k}$ ) are coded before the least important ones (with lower thresholds), there will be an ordering of wavelet coefficients resulting in progressively transmittable bit stream. The decoder can stop at any step and reconstruct the image that is best at that level. The image reconstructed at $T_{g}=0$ will be identical to the original image and hence results in lossless compression.

The main difference between our scheme and that proposed by [2] is in sending the significance map of the lattices. In [2], the array th is entropy coded and sent to the decoder. This impairs the performance of the scheme at higher thresholds. The bit budget that would be spent for resolutions at these thresholds is not worth. In our scheme, the significance information of lattices is sent only when it is required. This greatly increases the performance at higher thresholds (which is desired in a progressive transmission scheme). It has been shown in [8] that the above coding scheme performs better than that proposed by [2] and comparable to that of SPIHT for rectangular case. Moreover the scheme 
used here has an added advantage of simple implementation as compared to any interband coding techniques like SPIHT or EZW.

Coding of Binary Mask: The coding of binary mask is necessary because it contains the useful shape information. Rather than sending the whole binary mask we only send the boundary co-ordinates to the decoder after coding. We scan each row in the mask image and see which from which row the mask information starts and which row it ends, and send the starting and the ending row value. We see that each row cuts the boundary of the mask twice. We note down these values. We find the difference between successive column values and send this difference after entropy coding. The bit budget used in sending the shape information is of the order of $1 / 100$ bits per pixel (bpp).

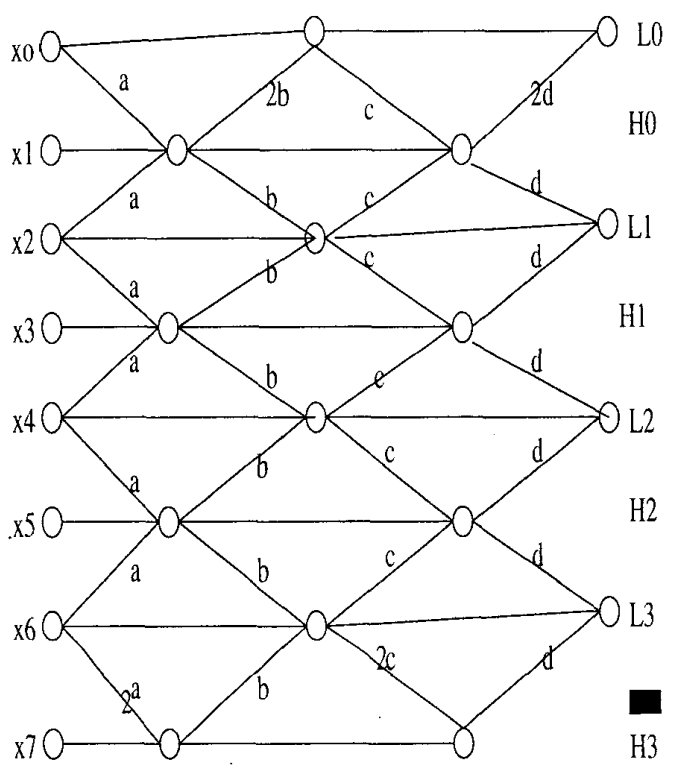

(a)

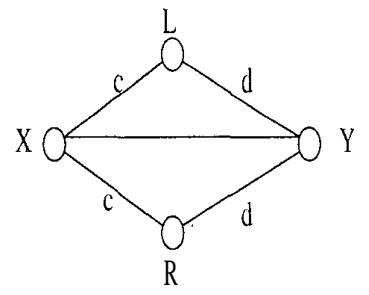

$Y=[(L+R) * d]+X$

(b)

Figure 4: Implementation of Integer Wavelet Transform
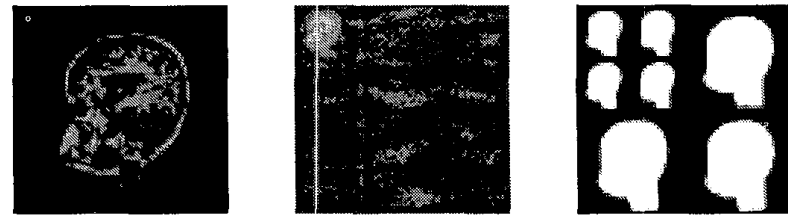

(a)

(b)

Figure 5: 2-D Shape Adaptive Integer Wavelet Transform (a) Original Image (b) 2-level Wavelet Decomposed Image (c) 2-level Decomposition of Mask

\section{EXTENSION TO REGION OF INTEREST BASED 3-D MR IMAGE COMPRESSION}

The above scheme can easily be extended to 3-D MR images. The motivation is to exploit intra and interframe correlation in these images. A common mask for a set of 2-D MR image slices (comprising 3-D MR data) is prepared by binary OR operation of individual masks of 2-D slices. This mask is used for coding all the MR slices.

We extend the above schemes by employing 3-D saparable SAIWT. A 1-D integer wavelet transform is applied is applied along the time axis for each pixel. This results in temporal low and high frequency images. Each of these images are then decomposed by saparable 2-D SAIWT as described in section 3 . We tiled the wavelet images by cuboids of size $\mathrm{v} x \mathrm{v} \times \mathrm{vt}(\mathrm{v}=4$ and $\mathrm{vt}=2)$. The algorithm in the previous section was used to obtain progressive bit stream. Fig $\mathbf{6}$ shows 1-level temporal and 1-level spatial Wavelet Decomposition which can be repeated to obtain higher level temporal and spatial wavelet decomposed images. Fig 7 shows the 2-level temporal and 2-level spatial 3-D shape adaptive transform of $8 \mathrm{MR}$ slices along with the decomposition of mask.

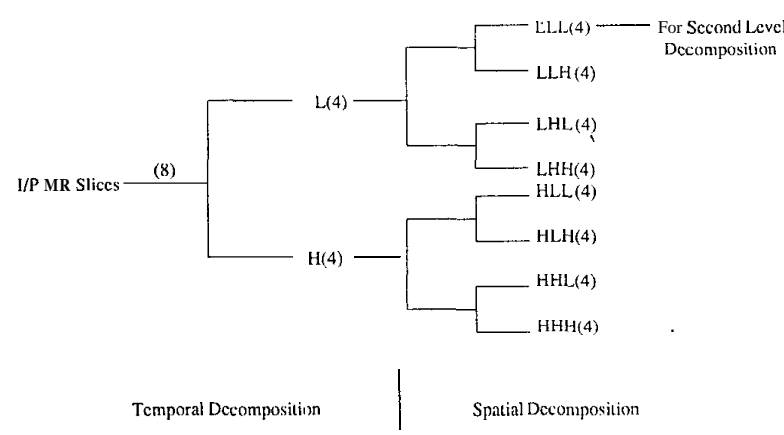

Figure 6: 1-level temporal and 1-level spatial Wavelet Decomposition; Note: The number of images are given in ()

\section{EXPERIMENTAL RESULTS}

We compare the performance of our Shape Adaptive Integer Wavelet based scheme with the conventional wavelet based scheme. We apply the above algorithms on 8-bit MR images provided by National Institute of Mental Health and Neuro 

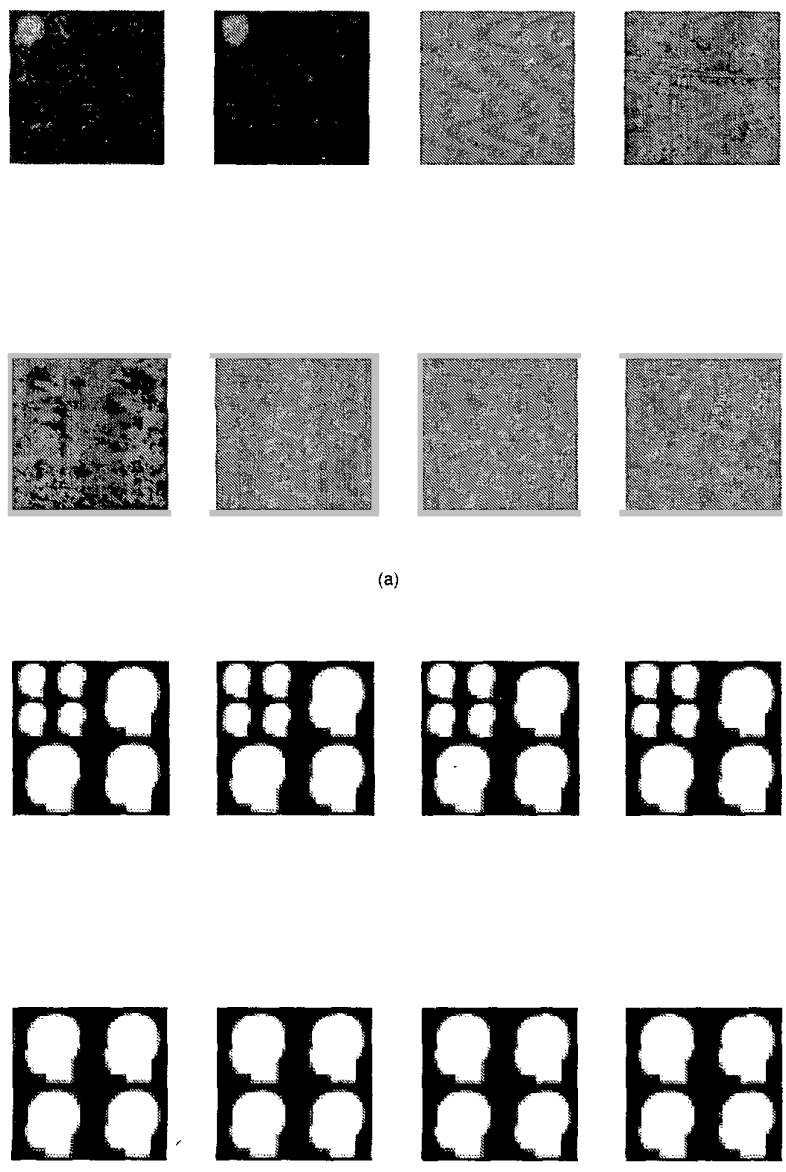

(b)

Figure 7: 3-D Shape Adaptive Integer Wavelet Transform of a set of 8 MR Image slices(a) 2-level temporal and 2-level spacial Wavelet Decomposed Image (b) 3-D Decomposition of Mask

Sciences (NIMHANS), Bangalore, India. We compare 2-D rectangular scheme wirh 2-D shape adaptive scheme and then 3-D rectangular with 3-D shape adaptive scheme.

The MR images are first preprocessed to remove background noise as explained in section 2. We take 2-level 2$\mathrm{D}$ integer wavelet decomposition of the preprocessed image. We use $\mathrm{v}=4$ for both the rectangular and shape adaptive schemes. For higher values of $\mathrm{v}$, the performance improves for higher thresholds, but at lower thresholds more coefficients are significant and hence more bits need to be spent for significance information. This reduces the performance at lower thresholds. We arrived at the value of 4 after experimenting with various values ofv. Figure 8 compares PSNR (peak signal to noise ratio) Vs Bit Rate of the two schemes. The performance of shape adaptive scheme is superior at all Bit Rates . On an average, the saving in the bit rate is $1.5 b p p$ at perfect reconstruction. Table-1 shows bit rate (in $b p p$ ) of

above 2-D schemes for different PSNRs for a representative image.

We implement 3-D algorithm on a group of $8 \mathrm{MR}$ images. The size of the images are $256 \mathbf{x} 256 \mathbf{x} 8$. The interframe thickness being Imm. We apply 2-level 3-D integer wavelet transform in both temporal and spatial directions. We use $\mathrm{v}=4$ and $\mathrm{vt}=2$. The saving in the bit rate is for 3-D shape adaptive case is 1.4bpp as compared to 3-D rectangular scheme at perfect reconstruction. Figure 9 compares PSNR (peak signal to noise ratio) Vs Bit Rate of the 3-D rectangular and shape adaptive schemes. The overall performance in the progressive transmission is impressive in extending the 2-D scheme to 3-D scheme. Table-2 shows bit rate (in bpp) of above 3-D schemes for different PSNRs for a set of 8 representative images.

Table: 1 PSNR in dB; Bit Rate in bpp: 2DRect : 2D Rectangular Scheme; 2Dshape : 2D Shape adaptive Scheme (including the shape information of the binary mask);

\begin{tabular}{l|l|l|l|l|l|l|l|l}
\hline PSNR & 16.3 & 22 & 27 & 32 & 38 & 44 & 50 & 100 \\
\hline 2DRect & .046 & .107 & .239 & .544 & 1.114 & 1.772 & 2.424 & 3.247 \\
\hline 2DShape & .051 & .101 & .220 & .501 & .959 & 1.346 & 1.560 & 1.687 \\
\hline
\end{tabular}

Table:2 PSNR in dB; Bit Rate in bpp; 3DRect : 3D Rectangular Scheme; 3Dshape : 3D Shape adaptive Scheme (including the shape information of the binary mask);

\begin{tabular}{l|c|l|l|l|l|l|l|c}
\hline PSNR & 16.3 & 22 & 27 & 32 & 38 & 44 & 50 & 100 \\
\hline 3DRect & .020 & .050 & .151 & .446 & 1.001 & 1.653 & 2.305 & 3.113 \\
\hline 3DShape & .029 & .056 & .157 & .447 & .916 & 1.300 & 1.566 & 1.712 \\
\hline
\end{tabular}

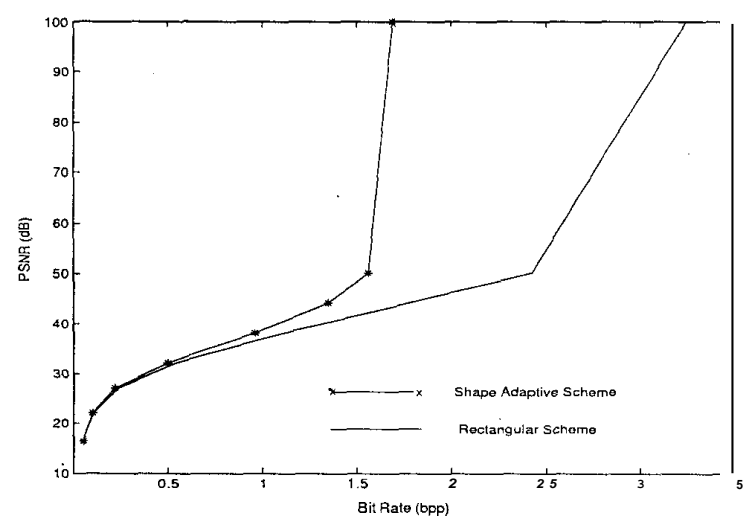

Figure 8: PSNR (in $\mathrm{dB}$ ) Vs Bit Rate (in bpp) performance curves of $2 \mathrm{D}$ schemes

\section{CONCLUSIONS}

We presented a new Shape Adaptive Integer Wavelet based lossless image compression scheme for 2-D and 3-D MR im- 


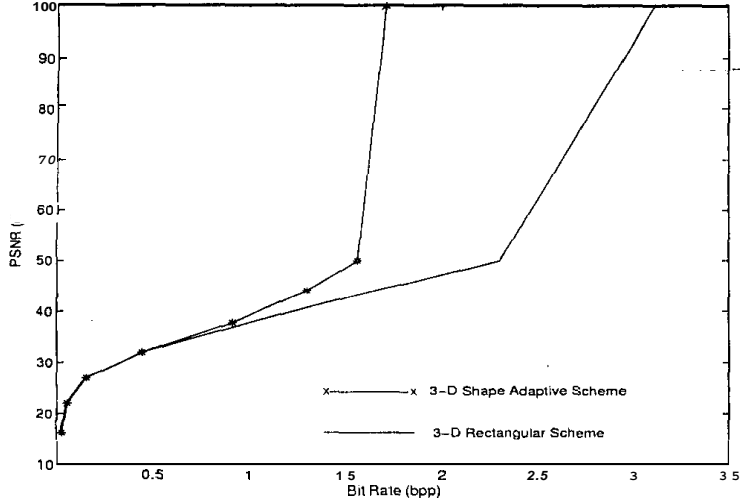

Figure 9: PSNR (in dB) Vs Bit Rate (in bpp) performance curves of 3D schemes

ages. This scheme exploits intraband correlation as opposed to interband correlation used by EZW and SPIHT schemes. This scheme is simple in implementation as compared to EZW and SPIHT algorithms. We got around $46 \%$ and $45 \%$ saving in Bit Rate for 2-D and 3-D respectively, at perfect reconstruction, using our new scheme as compared to conventional rectangular wavelet based schemes.

\section{ACKNOWLEDGEMENTS}

We thank Dr. P.N. Jayakumar, Prof and Head, Department of Neuroimaging and Interventional Radiology, NIMHANS, Bangalore for providing the images used in this work.

\section{REFERENCES}

[1] Marius Midtvik and Ingvil Hovig, "Universal Coding of MR images", IEEE Trans. Medical Imaging, vol.18, no.9, pp.795-800, September, 1999.

[2] Adrian Munteanu, Jan Cornelis, "Wavelet-Based Lossless Compression of Coronary Angiographic Images", IEEE Trans. Medical Imaging, vol.18, no.3, pp.272281.1999 .

[3] A.Said and W.pearlman, "A new fast and efficient image codec based on set partitioning in hierarchical trees", Circuits Syst. Video Technol., ,vol.6, pp 243-250, 1996.

[4] C.Christopoulos, J.Askelof and M.Larsson "Efficient methods for encoding regions of interest in upcoming JPEG2000 'still image coding standard", IEEE Signal Processing Lett.,, vol.7, pp 247-249, Sept. 2000.

[5] C.Christopoulos, J. Askelof and M.Larsson "Efficient region of interest encoding techniques in the upcoming JPEG2000 still image coding standard", Proc. Int. Conf. Image Processing (ICIP), vol.2, Vancouver, BC, Canada, Sept 10-13,2000,pp.41-44.
[6] J.M.Shapiro, "Embedded image coding using zerotrees of wavelet coefficients", IEEE Trans. Signal Process., vol.41, pp 3445-34.63, 1993.

[7] G.Menegaz and J.P.Thiran, "Lossy to Lossless ObjectBased Coding of 3-D MRI Data", IEEE Trans. Image Processing.,, vol.11, pp. 1053-1061, Sept. 2002.

[8] R. Srikanth and A.G. Ramakrishnan,"Wavelet based coding of 2-D and 3-D MR images", accepted for oral presentation IEEE Reigion 10 Conference (TENCON) 2003 ,

[9] Wim Sweldens" The Lifting Scheme: A custom-design construction of Biorthogonal Wavelets", Journal of Applied and computational Harmonic Analysis, vol.3, pp 186-200,1996.

[10] Guiwei Xing, Jin Li, Shipeng Li and Ya-Qin Zhang,"Arbitrarily Shaped Video Coding By Wavelet", IEEE Trans Circuits Syst. Video Technol.,, vol.11, pp 1135-1139. Oct.2001 